\title{
Comparative evaluation of scatter correction techniques in 3D positron emission tomography
}

\author{
Habib Zaidi \\ Division of Nuclear Medicine, Geneva University Hospital, 1211 Geneva 4, Switzerland \\ Received 19 June 2000 and in revised form 22 August 2000 / Published online: 12 October 2000 \\ (C) Springer-Verlag 2000
}

\begin{abstract}
Much research and development has been concentrated on the scatter compensation required for quantitative $3 \mathrm{D}$ positron emission tomography (PET). Increasingly sophisticated scatter correction procedures are under investigation, particularly those based on accurate scatter models and iterative reconstruction-based scatter compensation approaches. The main difference among the correction methods is the way in which the scatter component in the selected energy window is estimated. Monte Carlo methods provide further insight and might in themselves offer a possible correction procedure. Five scatter correction methods were compared in this study where applicable: the dual-energy window (DEW) technique, the convolution-subtraction (CVS) method, two variants of the Monte Carlo-based scatter correction technique (MCBSC1 and MCBSC2) and our newly developed statistical reconstruction-based scatter correction (SRBSC) method. These scatter correction techniques were evaluated using Monte Carlo simulation studies, experimental phantom measurements and clinical studies. Accurate Monte Carlo modelling is still the gold standard since it allows the separation of scattered and unscattered events and comparison of the estimated and true unscattered component. In this study, our modified version of Monte Carlo-based scatter correction (MCBSC2) provided a good contrast recovery on the simulated Utah phantom, while the DEW method was found to be clearly superior for the experimental phantom studies in terms of quantitative accuracy at the expense of a significant deterioration in the signal-to-noise ratio. On the other hand, the immunity to noise in emission data of statistical reconstruction-based scatter correction methods makes them particularly applicable to low-count emission studies. All scatter correction methods gave very good activity recovery values for the simulated 3D Hoffman brain phantom, which averaged within $3 \%$. The CVS and MCBSC1 techniques tended to overcorrect while SRBSC undercorrected for scatter in most regions of this phantom. It was concluded that all correction methods significantly improve the image quality and contrast compared to the case where no correction is applied. Generally, it was shown that the dif-
\end{abstract}

ferences in the estimated scatter distributions did not have a significant impact on the final quantitative results. The DEW method showed the best compromise between ease of implementation and quantitative accuracy, but entailed a significant deterioration in the signal-to-noise ratio.

Keywords: Positron emission tomography - Scatter correction - Monte Carlo - Phantoms

\section{Eur J Nucl Med (2000) 27:1813-1826}

DOI $10.1007 / \mathrm{s} 002590000385$

\section{Introduction}

Positron emission tomography (PET) offers the possibility of quantitative measurements of tracer concentration in vivo. However, there are several issues that must be considered in order to fully realise this potential. In practice, the measured line integrals must be corrected for a number of background and physical effects before or during reconstruction. These include dead-time correction, detector normalisation, subtraction of random coincidences, and attenuation and scatter corrections. To maintain accurate counting statistics, a relatively wide energy window is used owing to the finite energy resolution of the scintillation detectors employed in PET scanners - typically 10\%-30\% full-width at half-maximum (FWHM) at $511 \mathrm{keV}$. Therefore, rejection of scattered photons on the basis of energy discrimination has limited performance. Scatter qualitatively decreases contrast by misplacing events during reconstruction, and quantitatively causes errors in the reconstructed radioactivity concentrations by overestimating the actual activity. The impact of scatter in emission tomography generally depends on the photon energy, tomograph energy resolution and energy window settings, besides the object size, shape and chemical composition, and the source distribution. Many of these parameters are non-stationary, which implies a potential difficulty when developing proper 
scatter correction techniques. However, correction for scatter remains essential, not only for quantification but also for lesion detection and image segmentation [1].

Attenuation and scatter corrections in PET are now widely accepted by the nuclear medicine community as a vital component in the production of artefact-free, quantitative data. While attenuation correction is exact in PET, and limited mainly by the statistics of the acquired data, the problem of Compton scatter is far more complicated to solve [2]. Accurate scatter correction is one of the major problems facing quantitative 3D PET and still is an open question. Much research and development has been concentrated on the scatter compensation required for quantitative 3D PET. Increasingly sophisticated scatter correction procedures are under investigation, particularly those based on accurate scatter models and iterative reconstruction-based scatter compensation approaches. Most of the methods proposed so far attempt to estimate the scatter contamination and then remove it using either subtraction $[3,4,5]$ or convolution/deconvolution techniques $[6,7,8,9]$. An alternative to these methods would be to estimate the scatter component directly from the emission and transmission data $[10,11]$. Monte Carlo methods provide further insight and might in themselves offer a possible correction procedure [12, 13]. Development of scatter models that can be incorporated in statistical reconstruction (e.g. maximum likelihood-expectation maximisation or ML-EM) continues to be appealing; however, implementation must be efficient to be clinically applicable $[14,15]$. More recently, we have proposed a new scatter correction algorithm for fully 3D PET imaging [16]. The method is based on the hypothesis that the image corresponding to scattered events in the projection data consists of almost low-frequency components of activity distribution and that the low-frequency components will converge faster than the highfrequency ones in successive iterations of statistical reconstruction methods such as ML-EM or its accelerated version, the ordered subsets-expectation maximisation (OSEM) [17]. With the advent of faster computers and accelerated iterative reconstruction algorithms, different approaches to scatter compensation are receiving much attention.

The main difference between the correction methods is the way in which the scatter component in the selected energy window is estimated. The literature contains little information derived from systematic studies on the accuracy of different scatter correction techniques in 3D PET. A limited number of studies have reported the comparative evaluation of different scatter correction methods in both single-photon emission tomography (SPET) [18] and PET $[19,20]$ imaging. The primary concerns in relation to these methods are: (a) the scatter estimates may be inaccurate, leading to bias in the reconstructed images; and (b) the scatter compensation is often accompanied by a substantial increase in statistical noise. Moreover, many of the methods rely on calibration parameters that are sensitive to the imaging protocol and the physical performance of the tomograph, and may change for each patient.

In this study, an implementation of a modified version of the Monte Carlo-based scatter correction method (MCBSC2) and our newly proposed scatter correction approach based on statistical reconstruction (SRBSC) were evaluated, where applicable, against the original Monte Carlo-based scatter correction method (MCBSC1) [12] and more common correction methods (DEW, CVS) using Monte Carlo-simulated data, experimental phantom measurements and clinical studies. The goal of the study was to investigate the validity of the assumptions underlying the theoretical derivation of these methods and their potential in restoring the contrast, absolute activity quantification, recovery coefficient and signal-to-noise ratio (SNR).

\section{Materials and methods}

Scatter correction techniques

A number of scatter correction techniques have been proposed in 3D PET. They fall into four broad categories (see ref. [21] for a detailed description):

1. Energy window-based approaches

2. Convolution/deconvolution-based approaches

3. Approaches based on direct estimation of scatter distribution

4. Iterative reconstruction-based scatter compensation approaches

Different versions of the above methods have been successfully implemented for 3D PET. The algorithms compared in this paper are briefly described below.

The dual-energy window method (DEW). Multiple energy window methods were originally developed for SPET and have been in use for more than 15 years [22]. The development of the 3D acquisition mode in PET and improvements in the detector energy resolution have allowed the implementation of scatter correction based on the analysis of energy spectra. Several groups have investigated the potential of acquiring data in two [3, 4], three [5] and multiple [23] energy windows to develop corrections for scattering in 3D PET. The DEW algorithm used in this work is based on an implementation by the MRC PET methodology group at Hammersmith Hospital (UK) on the ECAT 953B scanner [4].

The convolution-subtraction method (CVS). Techniques based on convolution or deconvolution estimate the distribution of scatter from the standard photopeak data. The CVS method used in this work is based on an implementation by Bailey and Meikle [7]. The number of iterations used was four and the scatter fraction was tailored to the phantom or clinical study under consideration. It ranges from $30 \%$ in $3 \mathrm{D}$ brain scanning to $45 \%$ in whole-body oncological studies.

The original Monte Carlo-based scatter correction method (MCBSC1). The true scatter component (being an experimentally impossible measurement) can be accurately estimated using rigorous Monte Carlo simulations [24]. The possibility of using Monte Carlo simulation to calculate and correct for Compton scatter in 
3D PET was first suggested by Levin et al. [12]. Monte Carlo simulations require as input the voxelised source distribution as well as the corresponding distribution of attenuation coefficients. The method uses as an estimate of the true distribution, the non-scatter-corrected reconstructed 3D image volume for a simulated 3D PET acquisition, assuming that the number of events in each voxel accurately represents the number of annihilation photons originating at the corresponding location within the simulated body. The contours and attenuation coefficients of the different organs and tissues are obtained from a segmented transmission image derived from a short transmission scan.

The sinograms of simulated scattered and unscattered events are then created and used to estimate the scatter distribution for any given plane. As a result of the low detection probability, the Monte Carlo-generated data sets have a lower number of events than the measured data sets. Scaling of the simulated scatter distribution is therefore required to normalise the statistics of the simulated and the measured data. This scale factor is calculated as the ratio between the uncorrected emission sinograms and the total simulated emission sinogram (unscattered + scattered). Correction for scatter is performed by subtracting the estimated scatter distribution, $p_{s}{ }^{M C}$, multiplied by the calculated scale factor $(k)$ from the measured emission data set according to:

$P_{u}=P_{o}-k p_{s}^{M C}$

As proposed, the reconstructed emission image used as input to the simulations was not pre-corrected for scatter [12]. This results in an overestimation of the amount of scatter and blurring in the emission image due to mispositioning of events. The authors claim that the negative systematic errors introduced by the inclusion of scatter in the input image are less relevant than the statistical noise in the data sets. This standard method is referred to as MCBSC1 in this paper.

The modified Monte Carlo-based scatter correction method (MCBSC2). The contribution of scatter from out-of-FOV (field-ofview) activity is particularly significant in $3 \mathrm{D}$ whole-body scanning. To take this effect into account, activity from outside the FOV should be incorporated in the calculation of the scatter distribution [13]. For this purpose, data from several bed positions below and above the volume of interest should be made available as input to the Monte Carlo simulation. The effect of pre-correcting the projection data for scatter using an approximate method (e.g. CVS) was also investigated in this work. The data sets were precorrected for scatter with the CVS method and the reconstructed images were then used as input to the Monte Carlo simulator. This approach seems reasonable for a more accurate estimation of the true source distribution and is referred to below as MCBSC2, which can be distinguished from MCBSC1 by the modifications mentioned above.

Statistical reconstruction-based scatter correction (SRBSC). Reconstruction-based scatter compensation has received much attention in the literature during the past decade, and different algorithms belonging to this class of methods have been devised [14, $15,25,26]$. More recently, we proposed a new technique for scatter correction in 3D PET called statistical reconstruction-based scatter correction (SRBSC) [16]. Application of the method for scatter correction in SPET imaging was independently reported by another group [27]. The method is based on two hypotheses: (a) the scatter distribution consists mainly of a low-frequency component in the image, (b) the low-frequency components will converge faster than the high-frequency ones in successive iterations of statistical recon- struction methods (e.g. ML-EM). This non-uniform convergence property is further emphasised and demonstrated by Fourier analysis of the ML-EM algorithm [28, 29] and successive iterations of inverse Monte Carlo-based reconstructions [25].

The analysis of the response function to a line source in a water-filled cylinder revealed that the unscattered response kernels are limited to a small region around the location of the line source, while the scatter response kernels extend to a wide region outside the original activity distribution [16]. Therefore, the scatter components correspond to the low-frequency range in the source distribution. The properties mentioned above are exploited in the derivation of the SRBSC method for scatter correction. The low-frequency image is estimated using one iteration of the OSEM algorithm. The details and basic steps followed when implementing the algorithm are described elsewhere [16].

\section{Monte Carlo simulation studies}

The Monte Carlo simulator. The Eidolon Monte Carlo simulation package developed to simulate cylindrical 3D positron tomographs $[30,31]$ and available in the public domain since September 1999 was used in this work. Several phantom and clinical studies were considered to evaluate the accuracy of the Monte Carlo simulator in modelling the spatial distribution of detected events in the case of simple geometries as well as real and complex patterns of radioactivity distribution (see sections on phantom and clinical studies). Both phantom and clinical studies were simulated using the reconstructed emission data as an estimate of the activity distribution. A brain study was selected for illustration purposes. The voxelised source was represented by a single-volume PET study and out-of-FOV activity and scattering media were neglected. Transmission images were segmented in a uniform single soft tissue component in the case of brain imaging and two tissue components (lung and soft tissue) for the study in the thorax region.

Previously reported Monte Carlo validations of scatter correction techniques and related parameters have often been made using simple source and attenuating medium geometries and compositions. In addition to these evaluations, there is a need to investigate more clinically realistic source distributions to validate and compare scatter correction techniques. A comparison of the relative performance of different methods was performed using Monte Carlo simulated data sets of two digital phantoms. Simulated data for the DEW technique were not included owing to a technical difficulty in storing data acquired in two energy windows in the same file in CTI matrix format.

The Utah multi-compartment phantom. Phantom simulations of the Utah multi-compartment phantom were carried out. The compartments $\mathrm{A}, \mathrm{B}, \mathrm{C}$ and $\mathrm{D}$ of the phantom were filled with relative concentrations of 1:2:0:2, respectively (Fig. 1). In this study, the outer compartment $(\mathrm{E})$, which is generally used to provide activity from outside the FOV, was empty. A calculated attenuation correction was applied assuming a constant linear attenuation factor ( $\mu=0.096 \mathrm{~cm}^{-1}$ ) for water. The data sets were reconstructed using the reprojection algorithm (3DRP) [32] with a maximum opening angle corresponding to a ring index difference of 11. A Hanning filter having a cut-off frequency equal to 0.5 Nyquist was used for all studies.

The 3D Hoffman brain phantom. According to the user specifications, Eidolon can be used to simulate either simple analytical 
Fig. 1. Diagram of the Utah multi-compartment phantom used for quantitative analysis of the different correction techniques. The outer compartment (E) is generally used to provide activity from outside the fieldof-view

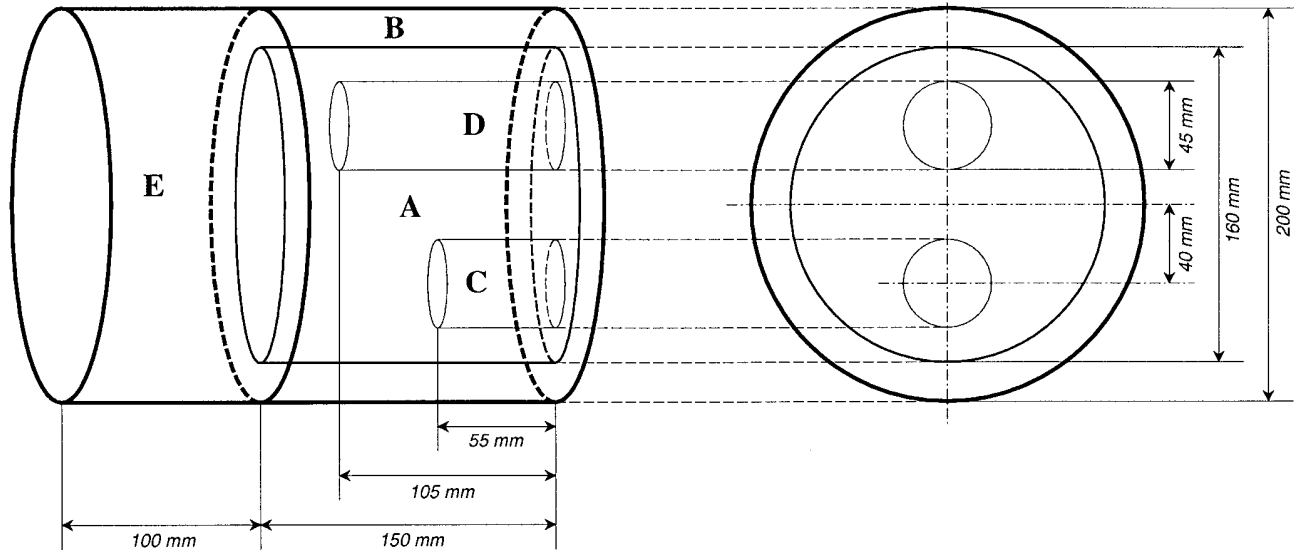

Experimental phantom studies

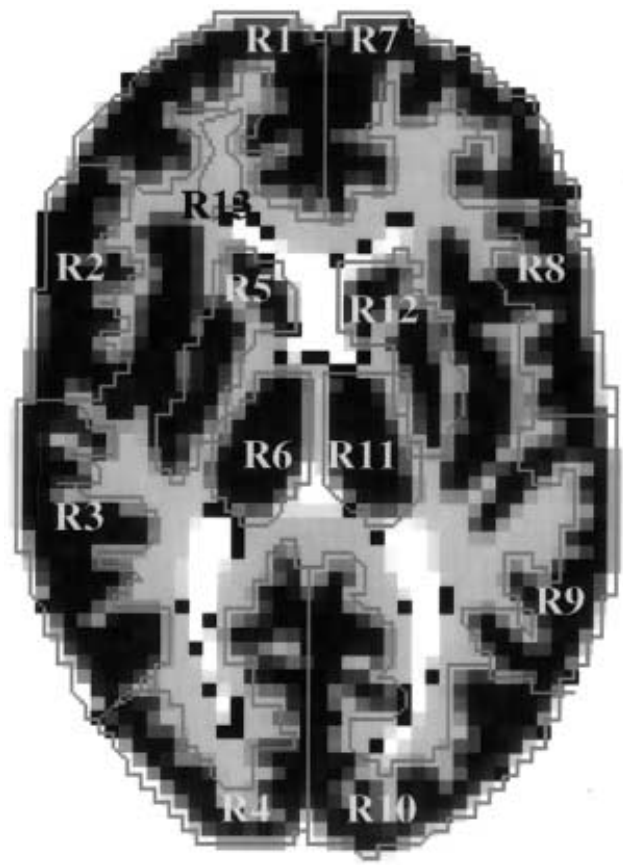

Experimental phantom studies were performed on the ECAT 953B scanner operated in 3D mode. Both the Utah and Hoffman 3D brain phantoms were scanned and analysed according to the same protocol used for the simulated data sets except that transmission studies were performed and used to correct the data sets for attenuation.

The physical Utah multi-compartment phantom. The Utah phantom was scanned with and without out-of-axial FOV activity. The outer compartment (E) was positioned just outside the FOV and was empty in the first acquisition, and then it was filled with an activity concentration equal to that in the inner cylinder (A) for the second acquisition. The compartments of the phantom (Fig. 1) were filled with relative concentrations of 1:1.68:0:2.03:0 (without out-of-FOV activity) and 1:1.68:0:2.03:1 (with out of-FOV activity) in A, B, C, D and E, respectively.

The physical 3D Hoffman brain phantom. A physical Hoffman $3 \mathrm{D}$ brain phantom [33] with a grey-to-white matter ratio of $4: 1$ was filled with fluorine-18, taking care to avoid the introduction of air bubbles. The data were acquired with moderate statistics (40 Mcounts).

\section{Clinical studies}

The different scatter correction algorithms were also tested on clinical data obtained on the prototype partial-ring cylindrical rotating positron tomograph (PRT-1) from Geneva University Hospital [34]. Cerebral and whole-body clinical studies were selected from the database and used for clinical evaluation of the scatter correction methods.

Cerebral scan study. A clinical brain scan of a patient who was referred to the Nuclear Medicine Division of Geneva University Hospital for evaluation was studied. A transmission measurement (15 min) using germanium-68 rotating rod sources was performed for attenuation correction purposes. The emission study started $30 \mathrm{~min}$ after intravenous injection of approximately $220 \mathrm{MBq}$ of ${ }^{18}$ F-fluorodeoxyglucose (FDG). The study was reconstructed by 3DRP with and without application of the different scatter correction techniques.

Clinical oncology study. An oncology study of a woman with a known stage T4 neoplasm of the breast and two metastases in the 
lung and the mediastinum was also considered. Data acquisition was performed at four bed positions covering an axial FOV of approximately $35 \mathrm{~cm}$ (four volumes, $15 \mathrm{~min} /$ volume). Transmission measurements were performed at each bed position $(10 \mathrm{~min} /$ position). The Monte Carlo package performs in principle a simulated 3D PET acquisition of a single bed position of the scanner axially positioned over the lungs and heart. Out-of-FOV activity was also incorporated by including three bed positions, one below and two above the simulation FOV. The study was reconstructed by 3DRP with and without application of the different correction techniques.

\section{Comparative assessment}

Comparative evaluation of different methods for scatter correction in $3 \mathrm{D}$ PET is inherently difficult owing to the multiplicity of the medical purposes for which the corrections may be studied. For any specific medical task, the evaluation should ideally be based on the performance of human observers [35]. However, this is costly and complex, since a reasonable number of experienced observers should be used to analyse many images under carefully controlled conditions, etc.

There is no single figure of merit that summarises algorithm performance, since performance ultimately depends on the diagnostic task being performed. Well-established figures of merit known to have a large influence on many types of task performance are used to assess image quality. These include calculations of the contrast and absolute concentrations measured in the different compartments of the Utah phantom, the signal-to-noise ratio and the recovery coefficient. With a few exceptions, most papers dealing with the evaluation of scatter correction techniques compare relative concentrations within different compartments of a given phantom with the background compartment serving as a reference. This approach was criticised by Bailey et al. [21] because it does not necessarily reflect the accuracy of the correction procedure and might bias the evaluation procedure. Hence, the evaluation reported here was performed in absolute terms. Different ROIs with diameters approximately equal to half the radius of the small cylinders were placed in the various sections of the Utah phantom and the mean counts/ROI in each section were determined for ideal, non-corrected and scatter corrected images. Contrast recovery (with an ideal value of $100 \%$ ) was calculated according to:

Contrast $=\frac{N_{B G}-N_{\text {cold }}}{N_{B G}} \times 100 \%$

where $N_{B G}$ is the count density in the background's ROI and $N_{\text {cold }}$ is the count density in the cold cylinder's ROI. The signal-to-noise ratio (SNR) was defined as the mean number of events divided by the standard deviation of pixel intensities in an ROI defined within the background region A of the Utah phantom (Fig. 1):

$S N R=\frac{\bar{N}_{R O I}}{\sigma_{R O I}}$

For the 3D Hoffman brain phantom, the activity recovery coefficients, defined as the percentage ratio of events in an ROI in the corrected images to events in the same ROI in the ideal images, were calculated according to:

Recovery $=\frac{N_{R O I}^{\text {corrected }}}{N_{R O I}^{\text {ideal }}} \times 100 \%$

where $N_{R O I}$ is the count density in the ROI.

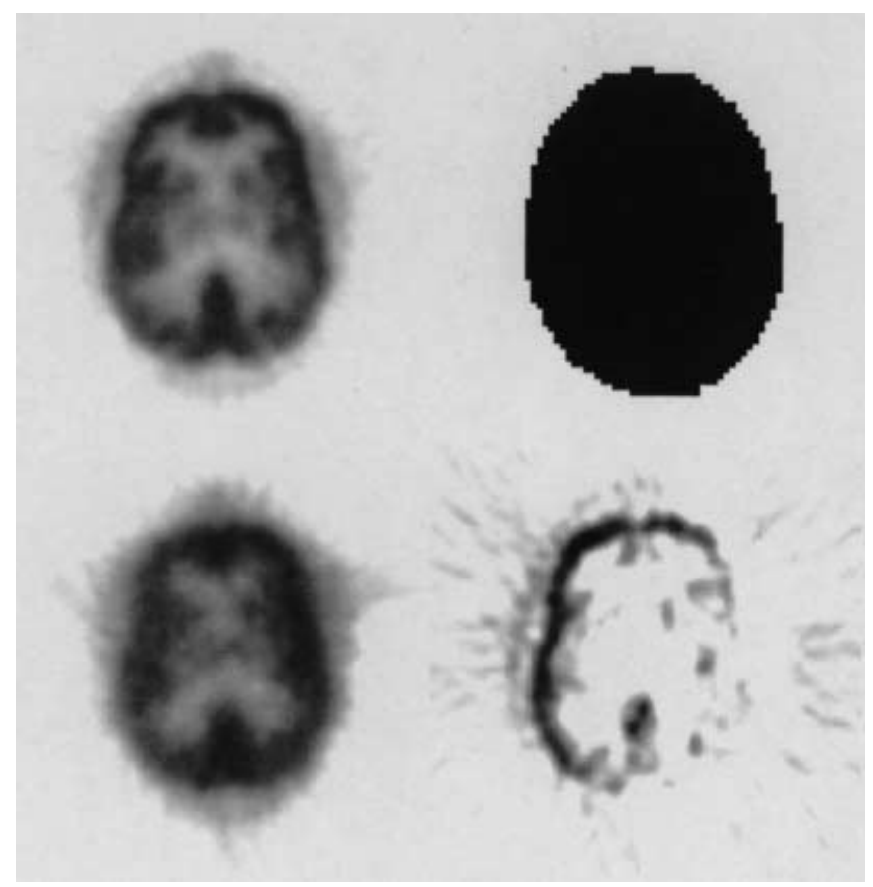

Fig. 3. Clinical cerebral study showing a representative slice of the original reconstructed radioactivity distribution (top left) and segmented transmission image (top right). The corresponding reconstructed image of the Monte Carlo simulated data (bottom left) and the normalised difference image between the original and the Monte Carlo reconstructed image, displayed using its own maximum intensity to display the differences in detail (bottom right), are also shown

\section{Results}

\section{Monte Carlo simulation studies}

Figure 3 illustrates the clinical cerebral study, showing a representative slice of the original reconstructed radioactivity distribution and segmented transmission image together with the corresponding reconstructed image of the Monte Carlo simulated data and the normalised difference between the original and the Monte Carlo reconstructed image. The simulated image was obtained by using as input to the Monte Carlo simulator the reconstructed 3D image volume as an estimate of the true distribution for a simulated 3D PET acquisition. There was good agreement between the two images, although the Monte Carlo data sets contained less statistics. The effect of precorrecting the first image estimate for scatter on the estimation of scatter distribution was extensively studied using different phantoms. Measured and simulated sinograms relative to the 31 direct planes were extracted from the 3D data sets. Profiles were obtained by averaging over all angular views. Figure 4 compares the measured and simulated sinograms for the clinical cerebral study (Fig. 3), showing the normalised profiles on a representative plane of the acquired sinograms. Note the good agreement between measured and simulated data when 


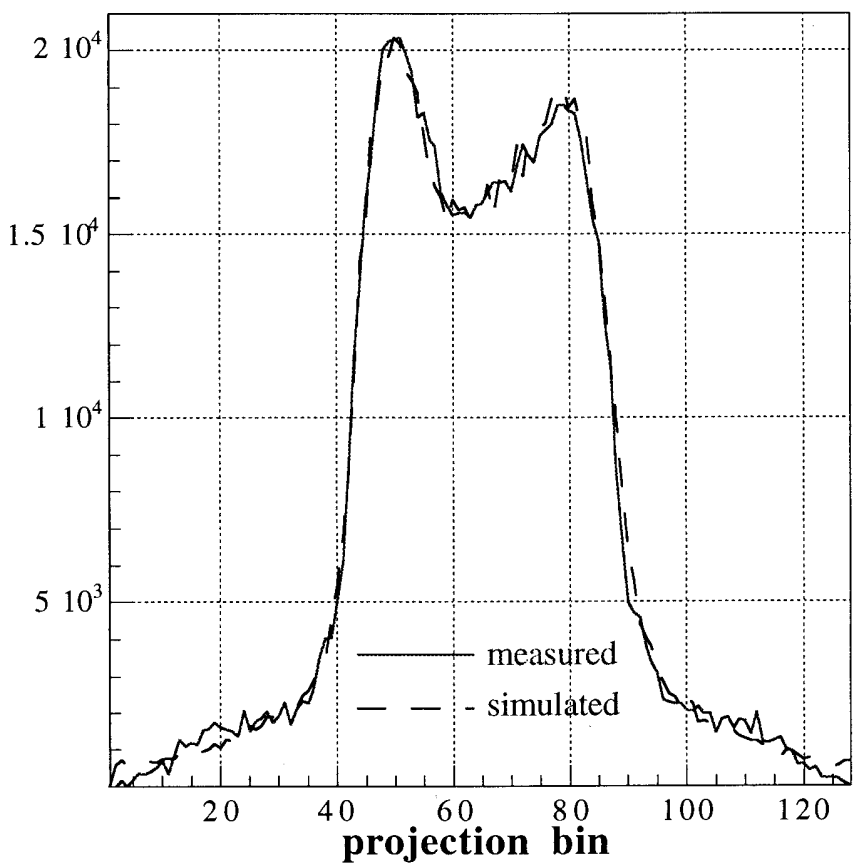

Fig. 4. Comparison between measured (solid line) and simulated (dashed line) profiles of a direct plane using scatter-corrected images as input to the Monte Carlo simulations of a clinical brain study the source distribution is corrected for scatter. Comparison of integral profiles of simulated scatter sinogram planes showed small differences between the two distributions. Normalised histogram profiles of the difference between the original sinogram and the simulated data are also shown in Fig. 5 for both simulations. It is worth pointing out that the amplitude of the error (difference in counts) is much smaller after scatter compensation.

The unscattered component in the simulated projection data was recorded and used as a reference to which the corrected projections using the different methods were compared. Figure 6 shows a comparison of a profile through a sinogram plane representing the true unscattered component as estimated by the simulations and by the correction procedures for both the Utah and 3D brain phantoms. Most scatter correction techniques give a reasonable estimation of the scatter component for the Utah phantom, which has a simple geometry and source distribution, and slightly overestimate the scatter component for the complex source distribution of the Hoffman 3D brain phantom. However, they successfully bring the activity to zero faster outside the object in both cases.

The reference image, the reconstructed image corresponding to unscattered events only, the image reconstructed after attenuation correction only (AC), and fi-
Fig. 5. Histogram profiles of the difference sinogram planes (\% error) using as input voxelised reconstructed images not corrected for scatter (left), and the same plane of Monte Carlosimulated sinograms using as input voxelised reconstructed images corrected for scatter (right), shown on the same axis scale. Note that the relative error is smaller after scatter compensation
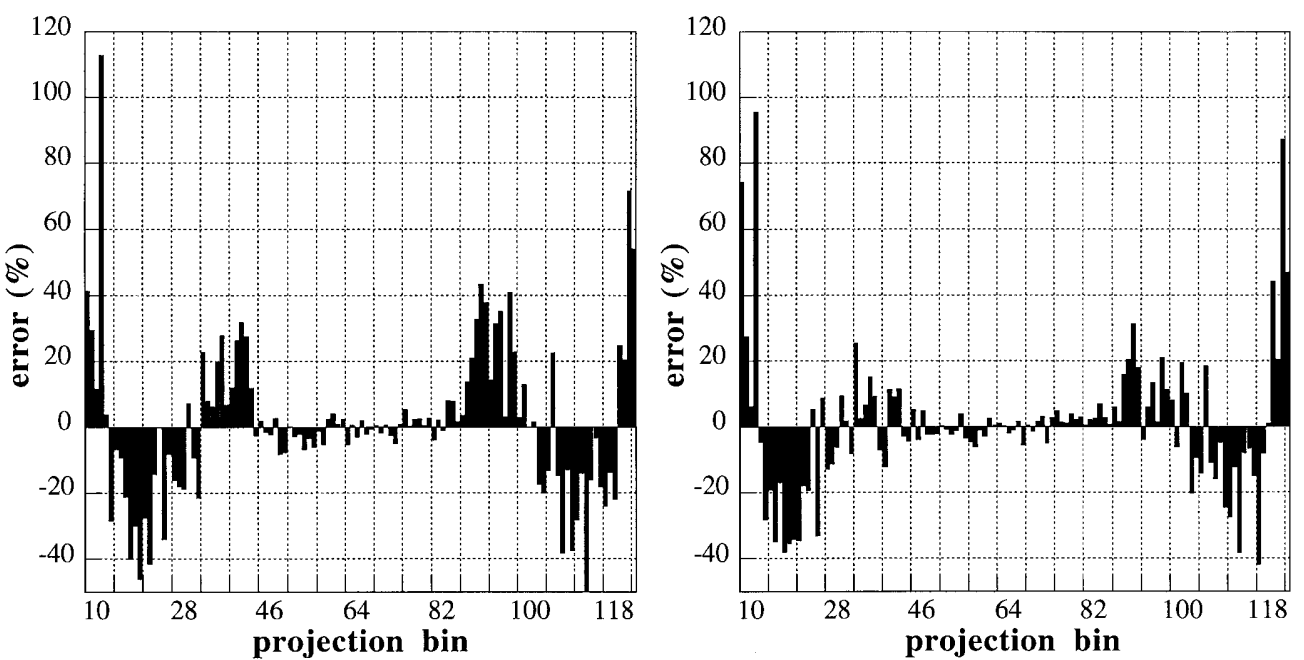

Table 1. Absolute concentrations and contrast measured in the different compartments of the simulated Utah phantom with attenuation correction only (AC) and after application of the different scatter correction techniques. The mean and standard deviations are shown. The signal-to-noise ratio (SNR) measured in the background (A) is also shown. The outer compartment (E) was not filled with activity

\begin{tabular}{|c|c|c|c|c|}
\hline \multirow{2}{*}{$\begin{array}{l}\text { Figure of merit } \\
\text { Case/compartment }\end{array}$} & \multicolumn{2}{|c|}{ Absolute concentration $(\mathrm{kBq} / \mathrm{ml})$} & \multirow{2}{*}{$\begin{array}{l}\text { Contrast (\%) } \\
\mathrm{C}\end{array}$} & \multirow{2}{*}{$\begin{array}{l}\mathrm{SNR} \\
\mathrm{A}\end{array}$} \\
\hline & $\mathrm{B}$ & $\mathrm{D}$ & & \\
\hline Reference image & 18.27 & 18.21 & 100 & $82.4 \pm 6.7$ \\
\hline Unscattered image & $19.78 \pm 0.44$ & $21.62 \pm 0.42$ & $78.2 \pm 5.3$ & $9.0 \pm 3.0$ \\
\hline $\mathrm{AC}$ & $24.71 \pm 0.53$ & $24.07 \pm 0.49$ & $63.8 \pm 5.2$ & $12.3 \pm 3.6$ \\
\hline CVS & $18.83 \pm 0.38$ & $20.84 \pm 0.39$ & $67.6 \pm 4.0$ & $7.7 \pm 2.1$ \\
\hline SRBSC & $19.23 \pm 0.39$ & $21.41 \pm 0.38$ & $69.4 \pm 4.5$ & $8.0 \pm 2.1$ \\
\hline MCBSC1 & $19.21 \pm 1.18$ & $21.31 \pm 1.07$ & $81.5 \pm 3.4$ & $6.4 \pm 1.4$ \\
\hline MCBSC2 & $19.60 \pm 0.94$ & $21.45 \pm 0.74$ & $88.8 \pm 6.5$ & $6.4 \pm 1.4$ \\
\hline
\end{tabular}


Fig. 6. Integral profiles through a sinogram plane of the simulated unscattered component and the scatter-corrected sinogram using the different correction techniques for the Utah phantom (a) and the Hoffman 3D brain phantom $(\mathbf{b})$

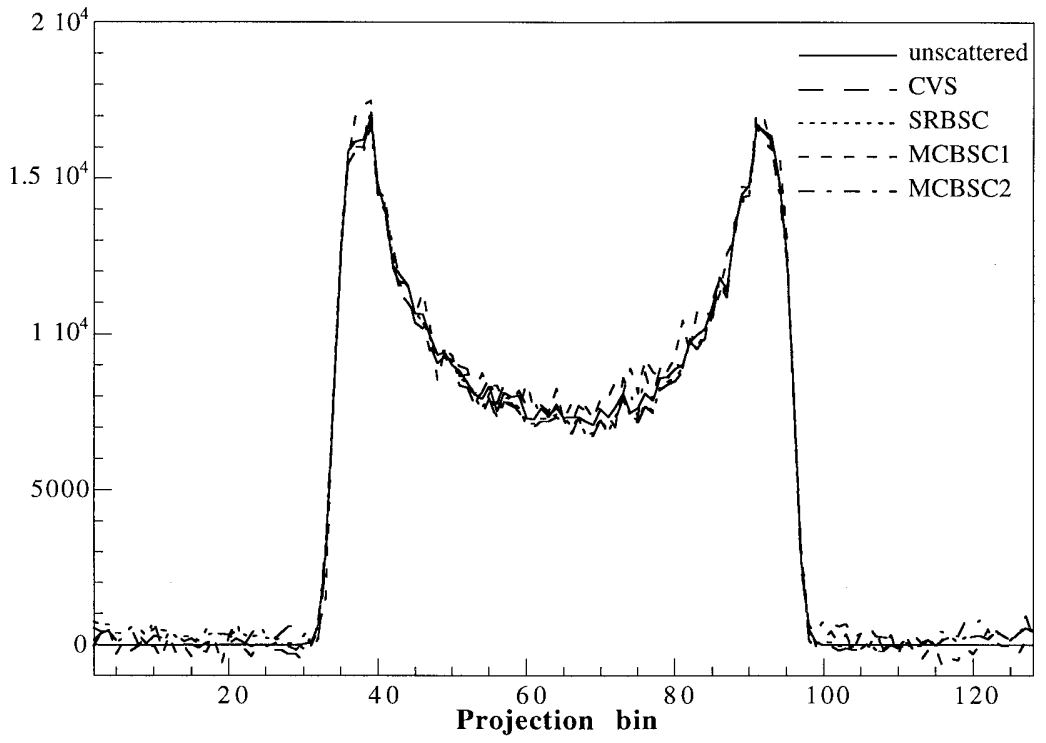

(a)

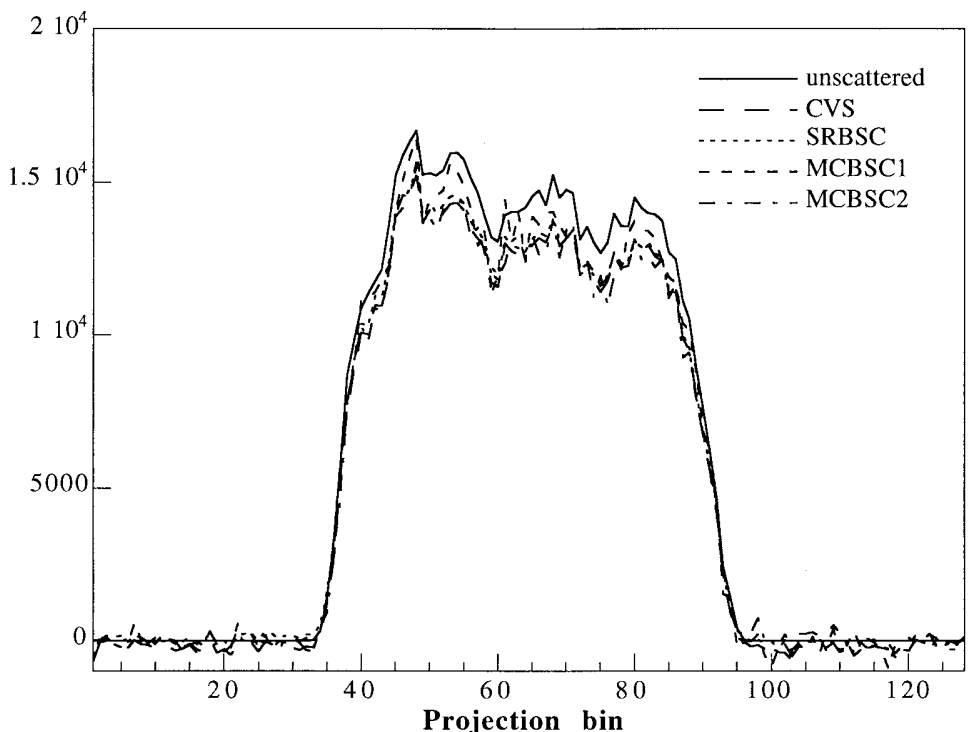

(b)
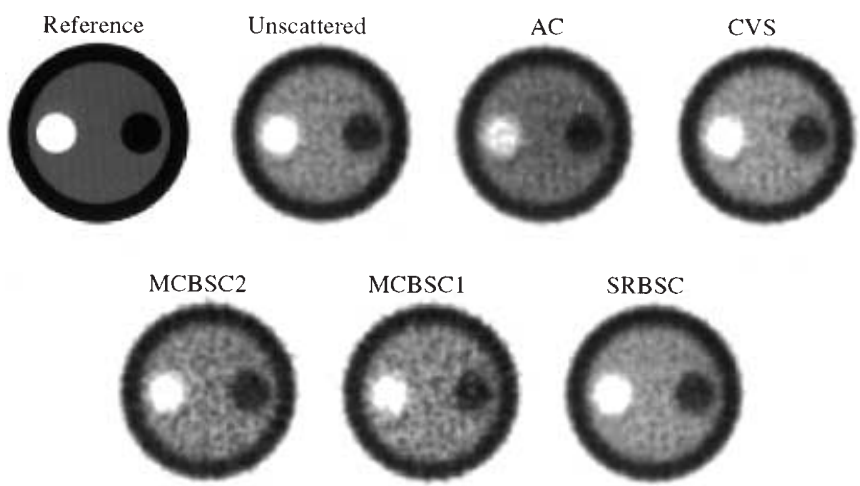

Fig. 7. Reconstructed images of the simulated Utah phantom. From top left, clockwise: the reference image used as input to the Monte Carlo simulations, the reconstructed image of unscattered events only, AC, CVS, SRBSC, MCBSC1 and MCBSC2 nally the images obtained after applying scatter subtraction using different methods for the simulated Utah phantom are illustrated in Fig. 7. All scatter correction techniques improve the quality of the images and allow a better definition of the cold cylinder (on the left) compared with the case where attenuation correction is applied alone; however, the images appear noisier when using Monte Carlo-based scatter correction techniques. Horizontal profiles through the images shown in Fig. 7 are illustrated in Fig. 8.

Table 1 shows the estimated contrast and absolute concentrations in the different compartments of the simulated Utah phantom for one distribution of activity before and after scatter correction is performed on the scans. Note that the reference image represents the actual statistical realisation of the ideal input image to the Monte Carlo simulation. An interesting observation from 
Table 2. Percentage recovery calculated in different structures of clinical interest in the Hoffman 3D brain phantom. The average and standard deviation (SD) are also shown
Fig. 8a, b. Horizontal profiles through the images shown in Fig. 7. a The reference image used as input to the Monte Carlo simulations (solid line), the reconstructed image of unscattered events only (dotted line), $\mathrm{AC}$ (short dashed line) and CVS (long dashed line). b SRBSC (dotted line), MCBSC1 (short dashed line) and MCBSC2 (long dashed line)

\begin{tabular}{|c|c|c|c|c|c|}
\hline \multirow[t]{2}{*}{ ROI } & \multicolumn{5}{|c|}{ Recovery (\%) } \\
\hline & $\mathrm{AC}$ & CVS & SRBSC & MCBSC1 & MCBSC2 \\
\hline $\mathrm{R} 1$ & $110.8 \pm 0.8$ & $98.6 \pm 0.1$ & $101.7 \pm 0.2$ & $97.8 \pm 0.5$ & $98.6 \pm 0.8$ \\
\hline $\mathrm{R} 2$ & $113.5 \pm 1.1$ & $98.0 \pm 0.1$ & $101.0 \pm 0.3$ & $98.3 \pm 1.2$ & $98.8 \pm 0.9$ \\
\hline R3 & $114.9 \pm 1.3$ & $97.6 \pm 0.3$ & $100.1 \pm 0.3$ & $97.8 \pm 0.8$ & $98.1 \pm 0.9$ \\
\hline $\mathrm{R} 4$ & $113.9 \pm 0.2$ & $98.1 \pm 0.3$ & $100.4 \pm 0.1$ & $96.9 \pm 1.2$ & $98.0 \pm 1.4$ \\
\hline R5 & $119.7 \pm 1.0$ & $96.7 \pm 0.6$ & $100.6 \pm 0.3$ & $97.7 \pm 0.8$ & $99.2 \pm 1.3$ \\
\hline R6 & $122.2 \pm 1.3$ & $97.1 \pm 4.3$ & $100.7 \pm 0.6$ & $99.2 \pm 1.7$ & $98.0 \pm 1.2$ \\
\hline R7 & $111.1 \pm 0.5$ & $98.3 \pm 0.3$ & $101.5 \pm 0.0$ & $98.3 \pm 1.1$ & $97.6 \pm 1.1$ \\
\hline R8 & $113.8 \pm 0.5$ & $97.8 \pm 0.4$ & $100.9 \pm 0.1$ & $98.5 \pm 0.9$ & $98.1 \pm 1.3$ \\
\hline R9 & $113.5 \pm 0.2$ & $97.8 \pm 0.4$ & $100.4 \pm 0.1$ & $97.5 \pm 1.1$ & $97.1 \pm 0.9$ \\
\hline $\mathrm{R} 10$ & $112.7 \pm 0.1$ & $98.4 \pm 0.2$ & $100.6 \pm 0.1$ & $98.6 \pm 1.3$ & $98.0 \pm 0.2$ \\
\hline $\mathrm{R} 11$ & $120.9 \pm 1.1$ & $96.9 \pm 0.2$ & $101.1 \pm 0.5$ & $97.3 \pm 2.3$ & $97.8 \pm 1.4$ \\
\hline $\mathrm{R} 12$ & $121.8 \pm 0.8$ & $96.8 \pm 0.4$ & $100.5 \pm 0.1$ & $97.3 \pm 0.6$ & $98.0 \pm 0.8$ \\
\hline $\mathrm{R} 13$ & $122.5 \pm 1.5$ & $97.1 \pm 1.0$ & $103.3 \pm 1.1$ & $97.7 \pm 0.9$ & $97.0 \pm 2.6$ \\
\hline Average & 116.2 & 97.6 & 101.0 & 97.9 & 98.0 \\
\hline SD & 4.4 & 0.6 & 0.8 & 0.6 & 0.6 \\
\hline
\end{tabular}

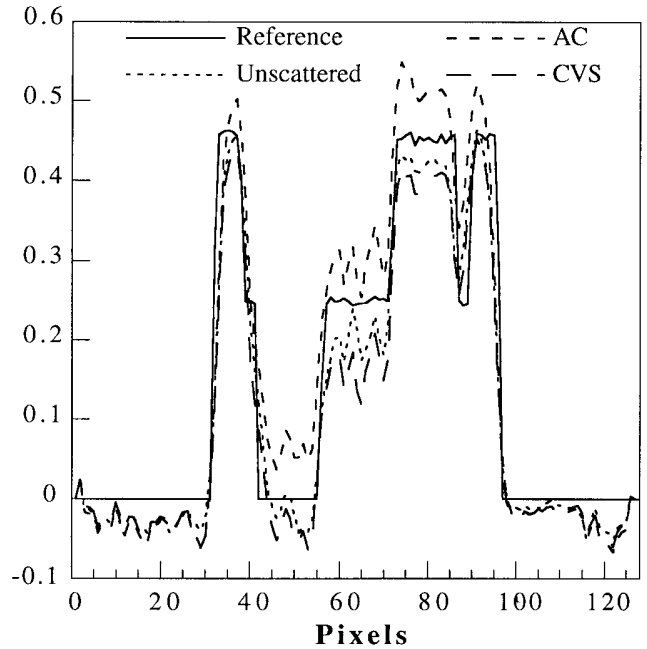

(a)
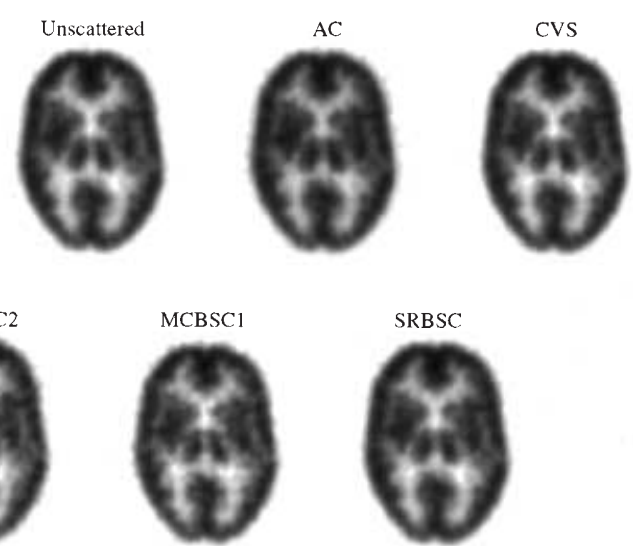

Fig. 9. Reconstructed images of the simulated Hoffman 3D brain phantom. From top left, clockwise: the reference image used as input to the Monte Carlo simulations, the reconstructed image of unscattered events only, AC, CVS, SRBSC, MCBSC1 and MCBSC2

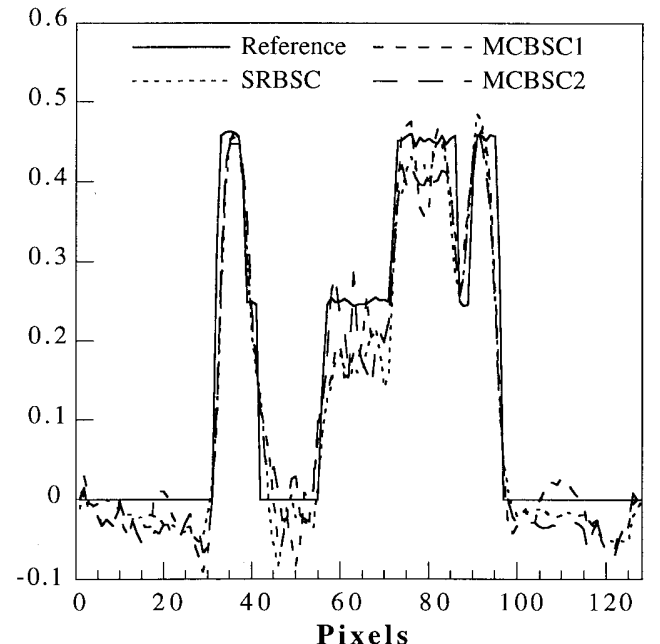

(b)

the analysis of the data presented in this table is that even in the reconstructed image corresponding to the unscattered component, the activity in the cold cylinder is not equal to zero as specified in the reference image used as input to the simulator. This difference may be attributed to the reconstruction algorithm, the finite spatial resolution of the simulated scanner and the resulting partial volume effect. The reduction in activity in the unscattered profile is more likely to be a reconstruction artefact from the filtered back-projection process and the reconstruction filter used. Scatter-corrected images have a poorer signal-to-noise ratio, which can be explained by the scatter subtraction process and the resulting reduction in the statistics of the acquired data sets; however, the quantitative accuracy is greatly improved. A remarkable enhancement of the quantitative accuracy in the different compartments is seen after scatter subtraction. Compared with the other methods, the MCBSC2 method 
allows better contrast recovery to be obtained in the cold compartment, at the expense of an increase in noise illustrated by a strong decrease in the signal-to-noise ratio. SRBSC slightly improves the SNR compared with the other techniques.

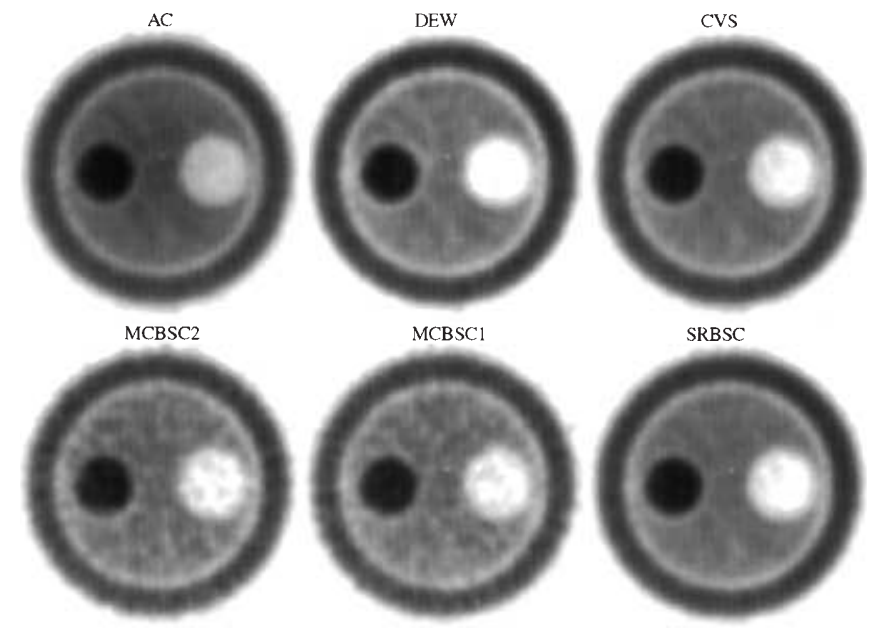

Fig. 10. Reconstructed images of the physical Utah phantom without activity in the outer compartment (out-of-FOV activity). The images shown are, from top left, clockwise: AC, DEW, CVS, SRBSC, MCBSC1 and MCBSC2
Reconstructions of the simulated 3D digital Hoffman brain phantom without corrections, after applying attenuation correction alone and when using the different scatter correction algorithms are shown in Fig. 9. The lowcount regions and structures are better recovered after scatter compensation. Table 2 shows the results of the quantitative evaluations of the percentage activity recovery using different correction techniques for the different ROIs, which cover important structures of the brain. All scatter correction methods give very good activity recovery values for the simulated 3D Hoffman brain phantom, which average within 3\%. The CVS and MCBSC1 techniques tend to overcorrect while SRBSC undercorrects for scatter in most regions of this phantom.

\section{Experimental phantom studies}

The reconstructed images of the physical Utah phantom without activity in the outer compartment (E) are shown in Fig. 10. Horizontal profiles through the images shown in Fig. 10 are illustrated in Fig. 11. The results of the quantitative analysis of the data are summarised in Table 3. All the methods improve the contrast compared to the case where no correction is applied. It can be seen that the DEW method predicts the contrast very accu-
Fig. 11. Horizontal profiles through the images shown in Fig. 9 illustrating: a AC (dashed line), DEW (solid line) CVS (dotted line), and b SRBSC (dashed line), MCBSC1 (solid line) and MCBSC2 (dotted line)

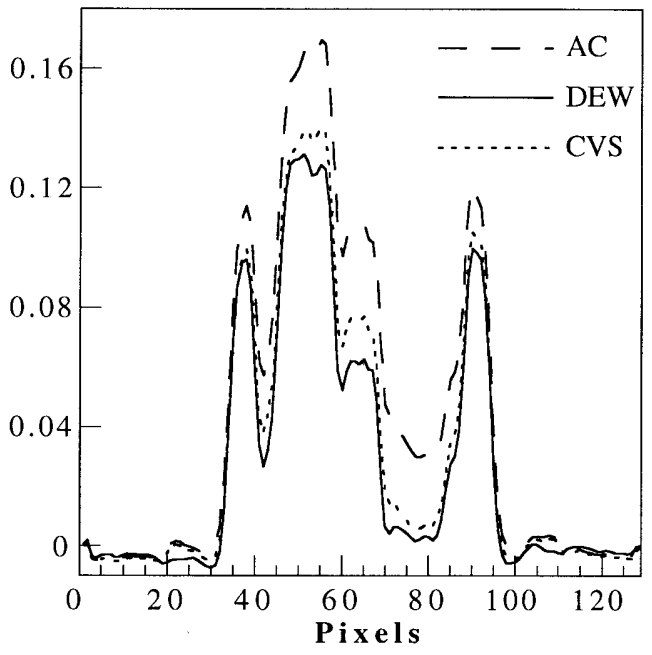

(a)

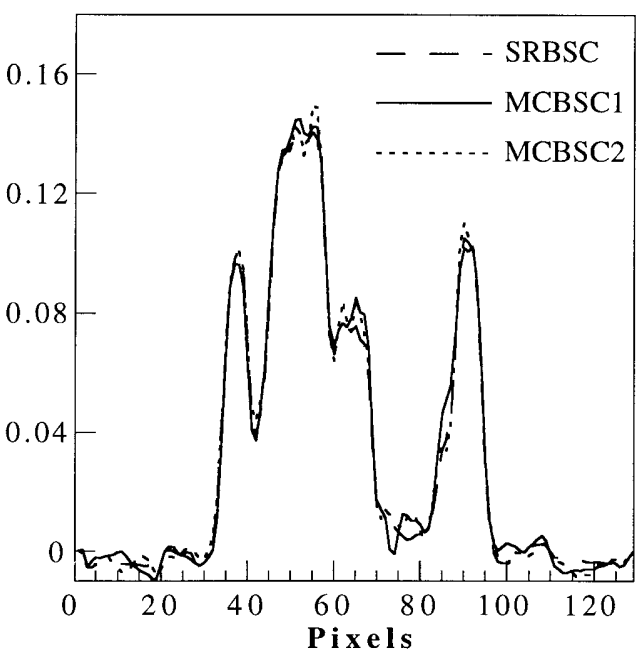

(b)
Table 3. Absolute concentrations and contrast measured in the different compartments of the scanned Utah phantom with attenuation correction only (AC) and after applying the different scatter correction techniques. The mean and standard deviations are shown. The signal-to-noise ratio (SNR) measured in the background (A) is also shown. The outer compartment (E) was empty

\begin{tabular}{|c|c|c|c|c|}
\hline \multirow{2}{*}{$\begin{array}{l}\text { Figure of merit } \\
\text { Case/compartment }\end{array}$} & \multicolumn{2}{|c|}{ Absolute concentration $(\mathrm{kBq} / \mathrm{ml})$} & \multirow{2}{*}{$\begin{array}{l}\text { Contrast (\%) } \\
\mathrm{C}\end{array}$} & \multirow{2}{*}{$\begin{array}{l}\text { SNR } \\
\mathrm{A}\end{array}$} \\
\hline & $\mathrm{B}$ & $\mathrm{D}$ & & \\
\hline Calibration concentration & 5.88 & 4.86 & 100 & - \\
\hline $\mathrm{AC}$ & $7.66 \pm 0.28$ & $5.31 \pm 0.17$ & $63.82 \pm 1.15$ & $21.91 \pm 5.17$ \\
\hline DEW & $6.05 \pm 0.23$ & $4.62 \pm 0.18$ & $91.63 \pm 1.84$ & $15.42 \pm 3.64$ \\
\hline CVS & $6.49 \pm 0.30$ & $4.68 \pm 0.23$ & $84.11 \pm 3.85$ & $18.79 \pm 4.54$ \\
\hline SRBSC & $6.52 \pm 0.30$ & $4.76 \pm 0.22$ & $86.26 \pm 3.95$ & $19.46 \pm 4.72$ \\
\hline MCBSC1 & $6.51 \pm 0.24$ & $4.81 \pm 0.21$ & $81.31 \pm 3.93$ & $9.74 \pm 2.43$ \\
\hline MCBSC2 & $6.55 \pm 0.27$ & $4.78 \pm 0.15$ & $85.02 \pm 1.76$ & $10.32 \pm 2.05$ \\
\hline
\end{tabular}


Table 4. Absolute concentrations and contrast measured in the different compartments of the scanned Utah phantom with attenuation correction only (AC) and after applying the different scatter correction techniques. The mean and standard deviations are shown. The signal-to-noise ratio (SNR) measured in the background (A) is also shown. The outer compartment (E) was filled with activity concentration equal to that in the background region

\begin{tabular}{|c|c|c|c|c|}
\hline \multirow{2}{*}{$\begin{array}{l}\text { Figure of merit } \\
\text { Case/compartment }\end{array}$} & \multicolumn{2}{|c|}{ Absolute concentration $(\mathrm{kBq} / \mathrm{ml})$} & \multirow{2}{*}{$\frac{\text { Contrast (\%) }}{\mathrm{C}}$} & \multirow{2}{*}{$\begin{array}{l}\text { SNR } \\
\mathrm{A}\end{array}$} \\
\hline & $\mathrm{B}$ & $\mathrm{D}$ & & \\
\hline Calibration concentration & 5.88 & 4.86 & 10 & - \\
\hline $\mathrm{AC}$ & $7.94 \pm 0.30$ & $5.47 \pm 0.15$ & $64.60 \pm 1.08$ & $19.04 \pm 4.69$ \\
\hline DEW & $6.14 \pm 0.21$ & $4.61 \pm 0.10$ & $95.74 \pm 2.09$ & $12.37 \pm 3.97$ \\
\hline CVS & $6.72 \pm 0.32$ & $4.82 \pm 0.20$ & $84.90 \pm 3.34$ & $16.24 \pm 4.33$ \\
\hline SRBSC & $6.76 \pm 0.32$ & $4.90 \pm 0.19$ & $86.78 \pm 3.30$ & $16.81 \pm 4.60$ \\
\hline MCBSC1 & $6.62 \pm 0.31$ & $4.72 \pm 0.24$ & $86.23 \pm 2.64$ & $9.78 \pm 3.37$ \\
\hline MCBSC2 & $6.77 \pm 0.24$ & $4.94 \pm 0.18$ & $86.33 \pm 1.54$ & $9.33 \pm 2.33$ \\
\hline
\end{tabular}
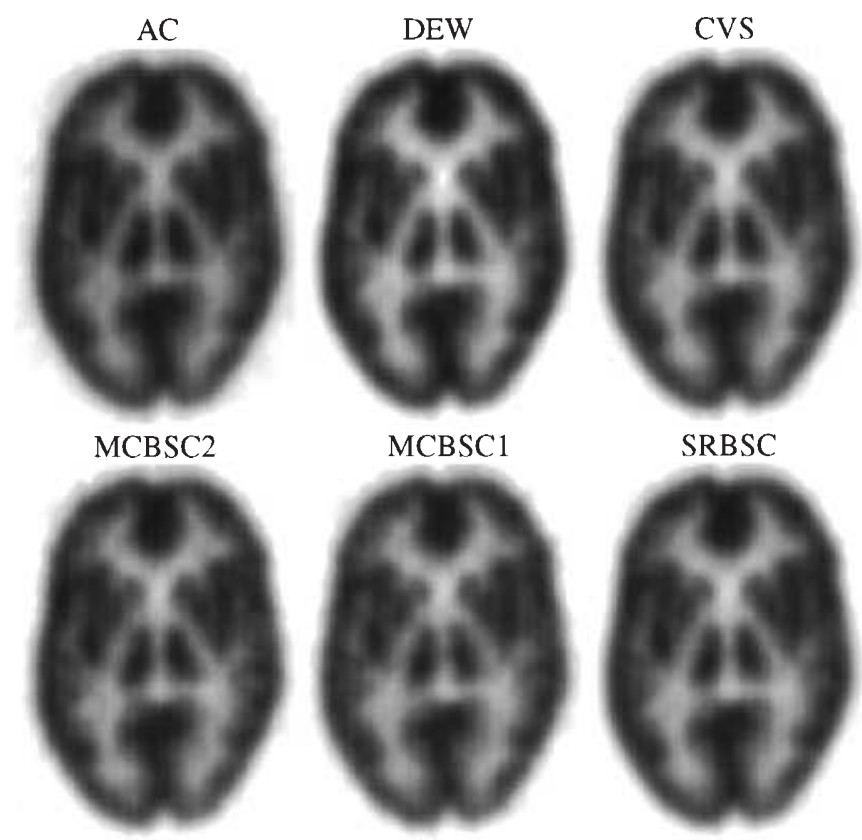

Fig. 12. Reconstructed images of the physical Hoffman 3D brain phantom. The images shown are, from top left, clockwise: AC, DEW, CVS, SRBSC, MCBSC1 and MCBSC2

rately as compared with the other methods but increases the noise significantly. On the other hand, SRBSC has a better signal-to-noise ratio and gives good contrast and quantitative accuracy in compartment A.

The results of the quantitative analysis of the Utah phantom with activity in the outer compartment (with out-of FOV activity) are summarised in Table 4. Again, the DEW predicts the contrast very accurately as compared with the other methods. Statistical noise was insignificantly changed by CVS and SRBSC whereas it was increased slightly by DEW and significantly by Monte Carlo-based approaches. The DEW method was more successful in correcting for scatter originating from outside the FOV. This was expected and has already been reported by other groups [19] since the CVS and SRBSC

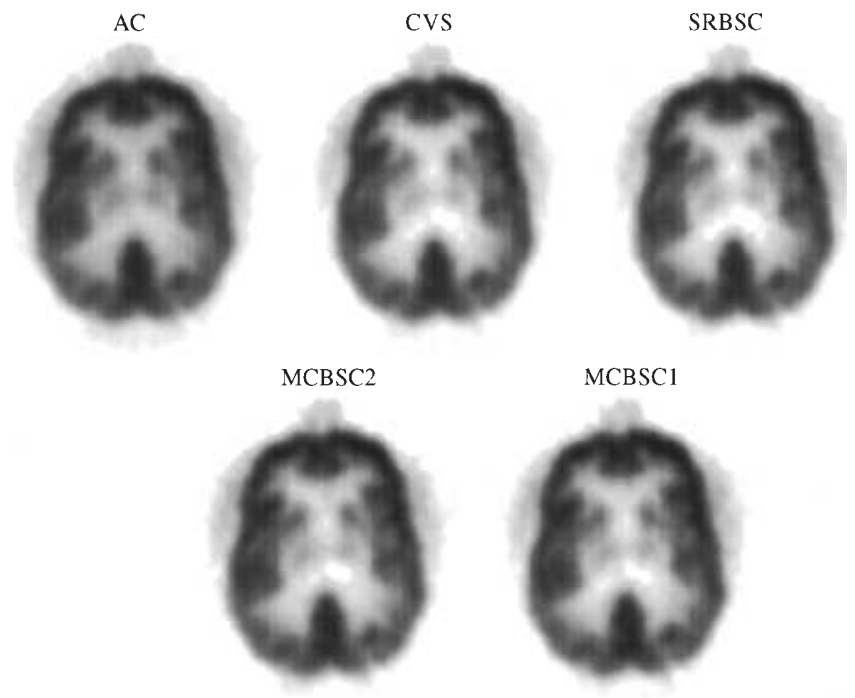

Fig. 13. Reconstructed images of a clinical brain study. From top left, clockwise: AC, CVS, SRBSC, MCBSC1 and MCBSC2

techniques estimate the scatter directly from measured data whereas the DEW method is sensitive to contributions from both in- and out-of-FOV activity.

The reconstructed images of the physical 3D brain phantom without and with the different correction techniques are shown in Fig. 12. It is hard to judge any major differences in the corrected images. The effect of scatter removal in areas where no activity is present is, however, clearly seen (e.g. cerebrospinal fluid or CSF). The DEW method better improves the contrast compared with the other methods.

\section{Clinical studies}

Figure 13 displays an image plane from the attenuationcorrected and scatter-corrected clinical brain study. Again, it is hard to judge any major differences in the corrected images. Nevertheless, the corrected images 
have a worse signal-to-noise ratio but better overall contrast between grey and white matter than the uncorrected original image. To better understand the effect of scatter correction on cerebral clinical data sets, a detailed analysis of the difference images between non-corrected and scatter-corrected projection data was performed (images not shown). It was demonstrated that the difference images resemble the attenuation-corrected images, particularly with respect to the contrast between the brain tissue and the air-filled sinuses. This is in agreement with the observations made by Bailey et al. [21]. Results obtained using the CVS approach for scatter correction in cerebral 3D PET scans (homogeneous attenuating region) have proven to be accurate $[7,20]$. Similar performances are obtained with our newly developed SRBSC approach, which is well adapted for this kind of study [16].

It should be noted that for the whole-body, especially at the level of the thorax, it was difficult to assess the effect of scatter correction on the images (not shown). However, the streak artefacts seen in the attenuation-corrected image only were significantly reduced after scatter subtraction by all techniques.

\section{Discussion}

This paper has focussed mainly on the use of Monte Carlo simulations, experimental measurements and clinical studies to characterise scatter correction algorithms in 3D PET imaging. Because of the finite energy resolution of block detectors, discrimination between unscattered and scattered events is not an easy task. Scattered coincidences add a background to the true coincidence distribution, decreasing contrast and causing the isotope concentrations to be overestimated. They also add statistical noise to the signal. The number of detected scattered events depends on energy window settings, the volume and attenuation characteristics of the object being imaged, and the tomograph's geometry. While the quantitative accuracy of 3D PET is limited mainly by attenuation and scatter corrections, it may also be influenced by the choice of the reconstruction algorithm. It is important to know both systematic and statistical errors in activity quantification when using different reconstruction algorithms.

To date most experimental investigations of scatter have been limited to simple homogeneous and symmetric phantoms. Modelling of imaging systems is best done with computer phantom models that match the gross parameters of individual patients. Recent three- and fourdimensional (dynamic) computer phantoms aim to achieve a reasonable compromise between ease of use, flexibility and accurate modelling of populations of patient anatomies, as well as attenuation and scatter properties and biodistributions of radiopharmaceuticals in the patients. Monte Carlo modelling has contributed to a better understanding of the physics of Compton scattering in PET. The development of efficient Monte Carlo simulation packages has allowed the study of scatter for realistic source distributions and attenuation maps and according to a variety of physical parameters [24]. Monte Carlo calculations are widely accepted as a powerful tool for study of the spatial characteristics of scatter for various phantoms [36]. In addition, the Monte Carlo method is uniquely able to provide information about multiple versus single scatter events. Knowledge gained from Monte Carlo simulations has contributed to the formulation and evaluation of new scatter correction techniques in 3D PET $[12,13,16]$.

Several approaches have been proposed for scatter correction in PET and a detailed description of these techniques can be found in Bailey et al. [21]. The convolution-subtraction approach can be applied to either projection data [7] or reconstructed images [8]. In the latter case, the scatter estimates are reconstructed and then subtracted from the non-corrected reconstructed images of the acquired data. Model-based scatter correction methods use both emission and transmission scans together with the physics of Compton scattering to estimate the scatter distribution [10, 11]. However, the above methods do not correct for scatter from outside the FOV. This effect can be directly taken into account by acquiring short, auxiliary scans adjacent to the axial volume being investigated. These extra data are naturally available in whole-body imaging. This implicitly assumes that the distribution of scatter from outside the FOV has the same shape as that of scatter from inside the FOV. The Monte Carlo-based scatter correction approach (MCBSC2) overcomes this limitation by incorporating an accurate model of scatter from outside the FOV. However, this method is impractical for isotopes with a short half-life or rapid uptake relative to the scanning interval. The somewhat disappointing results from the MCBSC2 method are thought to be due to the large statistical uncertainty after scatter subtraction. It is expected that the method will perform better for data acquired with high statistics.

Improvement in computer speed and recent advances in acceleration of reconstruction algorithms [17] have led to renewed interest in algorithms which incorporate scatter as part of the emission model. Iterative reconstruction-based scatter compensation has received considerable attention during the last decade. Earlier studies $[14,37]$ indicated that when scatter is modelled in iterative reconstruction, higher contrast and lower noise can be obtained than when the technique of scatter subtraction is used. In addition, model-based iterative reconstruction does not require patient-dependent parameterisation. One of the requirements of this method is to compute the scatter response function at each point in the attenuator with respect to each projection view. An efficient algorithm for scatter estimation was recently described in which the scatter spatial distribution is implemented as a spatially invariant convolution for points of 
constant depth in tissue [15]. The normal approach for implementation of a scatter model is to incorporate the scatter estimation directly in the transition matrix, although efficiency has been improved by utilising a dual matrix approach [38] in which scatter is incorporated in the forward projection step only. In this case, the transition matrix is considerably larger than is necessary if only attenuation and geometric factors are included, and computation is therefore slow since scatter is essentially recalculated and added in each iteration. It is worth pointing out that most of the research performed in this field is related to SPET imaging. Further development and validation of this class of algorithms in 3D PET is still required.

Scatter correction techniques can be evaluated using Monte Carlo simulation studies, experimental phantom measurements and clinical studies. Accurate Monte Carlo simulation is considered the gold standard since it allows separation of scattered and unscattered events and comparison of the estimated and true scatter components. A modification and extension to whole-body 3D imaging of a recently proposed Monte Carlo-based scatter correction method (MCBSC2) has been presented in this paper. A new scatter correction method called SRBSC [16] based on estimation of the low-frequency component corresponding to scatter events using OSEM reconstructions has also been evaluated against more common correction methods. Qualitative and quantitative comparisons of five scatter correction methods based on subtracting an estimated scatter component from the projection data have been performed where applicable. The aim was to compare the estimated scatter distributions with the true scatter distribution in the photopeak window.

It has been shown in a previous paper [16] that the parameterisation of the scatter response function can be obtained by Monte Carlo simulations and that the inherent assumptions in convolution-subtraction based approaches are still valid even in large objects like the thorax of a patient. Both CVS and SRBSC scatter correction approaches use a constant scatter response function and a global scatter fraction estimated from scanning standard objects equivalent to the size and shape of patients (in this case a line source in a uniform water-filled cylinder). It is believed that an accurate estimation of the scatter fraction is more important than modelling the spatially variant scatter functions [21]. The results presented in this paper seem to confirm this statement and suggest that these correction techniques are applicable to both cerebral 3D PET scans and more heterogeneously attenuating regions of the body such as the thorax. Nevertheless, different methods of non-stationary deconvolution have been proposed in the literature [39, 40]. Such an approach has some interesting features and we are investigating efficient implementations of a new method under development based on this model.

In this comparative study, the DEW method was found to be clearly superior in the experimental phantom studies in terms of quantitative accuracy, at the expense of a significant deterioration of the SNR (Tables 3,4). On the other hand, the immunity to noise in emission data of statistical reconstruction-based scatter correction methods makes them particularly applicable to lowcount emission studies. The DEW method is the easiest one to implement practically, but requires a system-specific calibration. A major disadvantage is that some commercial systems do not allow acquisition of coincidence events in separate windows (e.g. the GE-Advance).

Contribution of scatter from outside the FOV is a challenging issue that needs to be addressed with large axial FOV 3D PET scanners. Townsend et al. [19] investigated the effect of scatter from outside the FOV on the quantitative accuracy of parameter estimations in brain studies using carbon-11 flumazenil as a tracer. Based on phantom studies, the same conclusions as reported by us were drawn regarding the performance of the DEW approach for scatter correction as compared with the CVS technique. Nevertheless, the authors claim that this is not a major problem in cerebral studies and small axial FOV tomographs. The problem remains crucial for abdominal and thoracic studies, where contribution of scatter from outside the FOV is not negligible.

The Monte Carlo-based scatter correction procedure may be repeated iteratively to reduce systematic errors introduced by the presence of scatter in the input images and the low statistics in the simulated data. It is well recognised that Monte Carlo-based scatter correction may not be practical for clinical routine applications with common computing facilities available in PET centres. However, powerful multiple-processor parallel processing systems are becoming more accessible to the scientific community; therefore investigation and characterisation of such correction techniques and the effect of different approximations on their accuracy is worthwhile. To reduce computation time, it was proposed that coarse sampling of the input emission and segmented transmission images be used [12]. Although the method was developed for PET, we believe the basic principles of the method could also be applied to other scanner geometries, including dual-head coincidence cameras and the combined PET/CT system, as well as to other imaging modalities such as transmission CT and SPET. However, the success of such applications will depend on the efficient and accurate calculation of scatter responses from objects with non-uniform density and on the availability of suitable Monte Carlo simulators.

\section{Conclusion}

Accurate scatter correction would significantly improve the statistical power of conclusions drawn from research studies, improve diagnostic accuracy, and reduce the number of data sets discarded because of the non-quantitative nature of the data due to inaccurate scatter correc- 
tion. In summary, the quantitative accuracy and noise characteristics of different types of scatter correction techniques were investigated using simulations, phantom measurements and clinical studies. Generally, it was shown that the differences in the estimated scatter distributions did not have a significant impact on the final quantitative results.

The statistical errors in the scatter-corrected data have not been fully investigated. The error propagation has not been analysed and therefore no error limits have been given. In order to fully compare the scatter correction methods, further evaluation of the uncertainty in each correction needs to be made. It was concluded that all the methods improve the contrast compared with the case when no correction is applied and that an accurate modelling of the scatter component is essential for a proper scatter correction. The DEW method showed the best compromise between accuracy and ease of implementation. Further evaluation of the algorithms using simulated clinically realistic distributions in non-uniform attenuating regions like the thorax (e.g. the Zubal phantom) is still required.

Acknowledgements. This work was supported in part by the Swiss Federal Office for Education and Science under grant 96.193 within the European Esprit LTR project PARAPET (EP23493). The author gratefully thanks Dr. D. Bailey and Dr. T. Spinks for providing their scatter correction software, Dr K. Thielemans and D. Hogg for providing data sets acquired on the ECAT 953B, and Prof. A. Todd-Pokropek for fruitful discussions.

This paper is dedicated to the memory of my beloved father.

\section{References}

1. Zaidi H. Organ volume estimation using SPECT. IEEE Trans Nucl Sci 1996; 43: 2174-2182.

2. Thompson CJ. The problem of scatter correction in positron volume imaging. IEEE Trans Med Imag 1993; 12: 124-132.

3. Bendriem B, Trebossen R, Frouin V, Syrota A. A PET scatter correction using simultaneous acquisitions with low and high lower energy thresholds. In: Proc. IEEE Med. Imag. Conf. Piscataway, N.J.: IEEE; 1993; 3: 1779-1783.

4. Grootoonk S, Spinks TJ, Sashin D, Spyrou NM, Jones T. Correction for scatter in 3D brain PET using a dual energy window method. Phys Med Biol 1996; 41: 2757-2774.

5. Shao L, Freifelder R, Karp JS. Triple energy window scatter correction technique in PET. IEEE Trans Med Imag 1994; 4: 641-648.

6. Bergstrom M, Eriksson L, Bohm C, Blomqvist G, Litton J. Correction for scattered radiation in a ring detector positron camera by integral transformation of the projections. J Comput Assist Tomogr 1983; 7: 42-50.

7. Bailey DL, Meikle SR. A convolution-subtraction scatter correction method for 3D PET. Phys Med Biol 1994; 39: 411-424.

8. Lercher MJ, Wienhard K. Scatter correction in 3D PET. IEEE Trans Med Imag 1994; 13: 649-657.

9. Cherry S, Huang SC. Effects of scatter on model parameter estimates in 3D PET studies of the human brain. IEEE Trans Nucl Sci 1995; 42: 1174-1179.
10. Ollinger JM. Model-based scatter correction for fully 3D PET. Phys Med Biol 1996; 41: 153-176.

11. Watson CC, Newport D, Casey ME, deKemp A, Beanlands RS, Schmand M. Evaluation of simulation-based scatter correction for 3-D PET cardiac imaging. IEEE Trans Nucl Sci 1997; 44: 90-97.

12. Levin CS, Dahlbom M, Hoffman EJ. A Monte Carlo correction for the effect of Compton scattering in 3-D PET brain imaging. IEEE Trans Nucl Sci 1995; 42: 1181-1188.

13. Levin CS, Tai Y-C, Hoffman EJ, Dahlbom M, Farquhar TH. Removal of the effect of Compton scattering in 3-D PET whole-body positron emission tomography by Monte Carlo. In: Proc. IEEE Med. Imag. Conf. Piscataway, N.J.: IEEE; 1996; II: 1050-1054.

14. Beekman FJ, Kamphuis C, Frey EC. Scatter correction methods in 3D iterative SPECT reconstruction: a simulation study. Phys Med Biol 1997; 42: 1619-1631.

15. Hutton BF, Baccarne V. Efficient scatter modelling for incorporation in maximum likelihood reconstruction. Eur $\mathrm{J} \mathrm{Nucl}$ Med 1998; 25: 1658-1665.

16. Zaidi H. Statistical reconstruction-based scatter correction: a new method for 3D PET. Conf. Proc. of the World Congress on Medical Physics and Biomedical Engineering 2000 (available at http://dmnu-pet5.hcuge.ch/).

17. Hudson HM, Larkin RS. Accelerated image reconstruction using ordered subsets of projection data. IEEE Trans Med Imag 1994; 13: 601-609.

18. Buvat I, Rodriguez-Villafuerte M, Todd-Pokropek A, Benali $\mathrm{H}$, Di Paola R. Comparative assessment of nine scatter correction methods based on spectral analysis using Monte Carlo simulations. J Nucl Med 1995; 36: 1476-1488.

19. Townsend DW, Price JC, Mintun MA, Kinahan PE, Jadali F, Sashin D, Simpson N, Mathis CA. Scatter correction for brain receptor quantitation in 3D PET. In: Myers R, Cunningham VJ, Bailey DL, Jones T, eds. Quantification of brain function using PET. San Diego: Academic Press; 1996: 76-81.

20. Sossi V, Oakes TR, Ruth TJ. A phantom study evaluating the quantitative aspect of 3D PET imaging of the brain. Phys Med Biol 1998; 43: 2615-2630.

21. Bailey DL et al. Quantitative procedures in 3D PET. In: Bendriem B, Townsend DW, eds. The theory and practice of 3D PET. The Netherlands: Kluwer Academic; 1998: 55-109.

22. Jaszczak RJ, Greer KL, Floyd CE, Harris CC, Coleman RE. Improved SPECT quantification using compensation for scattered photons. J Nucl Med 1984; 25: 893-900.

23. Bentourkia M, Msaki P, Cadorette J, Lecompte R. Energy dependence of scatter components in multispectral PET imaging. IEEE Trans Nucl Sci 1995; 42: 138-145.

24. Zaidi H. Relevance of accurate Monte Carlo modeling in nuclear medical imaging. Med Phys 1999; 26: 574-608.

25. Floyd CE, Jaszczak RJ, Greer KL, Coleman RE. Inverse Monte Carlo as a unified reconstruction algorithm for ECT. $J$ Nucl Med 1986; 27: 1577-1585.

26. Kadrmas DJ, Frey EC, Karimi SS, Tsui BMW. Fast implementations of reconstruction-based scatter compensation in fully $3 \mathrm{D}$ SPECT image reconstruction. Phys Med Biol 1998; 43: 857-874.

27. Liu Z, Obi T, Yamaguchi M, Ohyama N. Fast estimation of scatter components using the ordered subsets expectation maximization algorithm for scatter compensation. Opt Rev 1999; 6: 415-423.

28. Pan TS, Yagle AE. Numerical study of multigrid implementations of some iterative image reconstruction algorithms. IEEE Trans Med Imag 1991; 10: 572-588. 
29. Tanaka E. A fast reconstruction algorithm for stationary positron emission tomography based on a modified EM algorithm. IEEE Trans Med Imag 1987; 6: 98-105.

30. Zaidi H, Herrmann Scheurer A, Morel C. An object-oriented Monte Carlo simulator for 3D positron tomographs. Comput Methods Programs Biomed 1999; 58: 133-145.

31. Zaidi H, Labbé C, Morel C. Implementation of an environment for Monte Carlo simulation of fully 3D positron tomography on a high-performance parallel platform. Parallel Comput 1998; 24: 1523-1536.

32. Kinahan PE, Rogers JG. Analytic 3D image reconstruction using all detected events. IEEE Trans Nucl Sci 1989; 36: 964-968.

33. Hoffman EJ, Cutler PD, Digby WM, Mazziotta JC. 3-D phantom to simulate cerebral blood flow and metabolic images for PET. IEEE Trans Nucl Sci 1990; 37: 616-620.

34. Townsend DW, Wensveen M, Byars LG, et al. A rotating PET scanner using BGO block detectors: design, performance and applications. J Nucl Med 1993; 8: 1367-1376.

35. Swets JA, Pickett RM. Evaluation of diagnostic systems: methods from signal detection theory. New York: Academic Press, 1982.
36. Adam L-E, Karp JS, Brix G. Investigation of scattered radiation in 3D whole-body positron emission tomography using Monte Carlo simulations. Phys Med Biol 1999; 44: 28792895.

37. Frey EC, Tsui BMW. A fast projector-backprojector pair modeling the asymmetric, spatially varying scatter response function for scatter compensation in SPECT imaging. IEEE Trans Nucl Sci 1993; 40: 1192-1197.

38. Kamphuis C, Beekman FJ, van Rijk PP, Viergever MA. Dual matrix ordered subsets reconstruction for accelerated 3D scatter compensation in single-photon emission tomography. Eur $J$ Nucl Med 1998; 25: 8-18.

39. Ljungberg M, Strand S-E. Scatter and attenuation correction in SPECT using density maps and Monte Carlo simulated scatter functions. J Nucl Med 1990; 31: 1560-1567.

40. Welch A, Gullberg GT, Christian PE, Datz FL, Morgan HT. A transmission-map-based scatter correction technique for SPECT in inhomogeneous media. Med Phys 1995; 22: 1627 1635 . 